\title{
Restauración en laderas mineras: una oportunidad para estudiar la sucesión ecológica en procesos de pendiente
}

\author{
Restoration in mining hillsides: \\ an opportunity to study the ecological succession in slope processes
}

López-Marcos, D. ${ }^{1,2, *}$; Turrión Nieves, M.B., $;$ Martínez-Ruiz, C.,3

${ }^{1}$ Área de Ecología - Departamento de Ciencias Agroforestales.

ETSIIAA de Palencia. Universidad de Valladolid.

²Área de Edafología y Química Agrícola - Departamento de Ciencias Agroforestales. ETSIIAA de Palencia. Universidad de Valladolid.

${ }^{3}$ Instituto Universitario de Investigación en Gestión Forestal Sostenible UVa - INIA 


\section{Resumen}

En restauración de los taludes de minas de carbón se genera una elevada heterogeneidad edáfica que determina importantes diferencias en la comunidad vegetal. Un mayor conocimiento sobre los procesos ecológicos involucrados es indispensable para garantizar el éxito de la revegetación en ambientes degradados. Este trabajo evalúa la influencia de la variabilidad espacial de parámetros edáficos y topográficos sobre la distribución de la vegetación en una ladera de una mina de carbón restaurada. La ladera, con fuerte pendiente y ubicada en Guardo (Palencia), se hidrosembró en octubre de 2000. En primavera de 2011 se establecieron seis transectos paralelos al gradiente topográfico, tres en una comunidad de pastizal y tres en otra de matorral. Se caracterizaron los suelos y la vegetación a lo largo del gradiente topográfico. La vegetación se clasifico por familias y por ciclo de vida. Se observó un gradiente en la distribución de la vegetación en respuesta a la altitud, al aumento de la capacidad de retención de agua y del espesor del suelo, y a la disminución de la ratio carbono fácilmente oxidable/carbono total. El pastizal ocupó la parte más alta de la ladera y el matorral la más baja. Se modelizó la respuesta de la vegetación a lo largo del gradiente altitudinal, y se observó que leñosas y leguminosas dominaban las partes bajas de la ladera mientras que las partes más elevadas estaban ocupadas por gramíneas y anuales. Los resultados sugieren que el diseño de las laderas para la revegetación debe considerar las condiciones topográficas locales.

Palabras clave: Gradiente topográfico, Restauración de espacios degradados, Relación planta-suelo, Sucesión vegetal.

\section{Abstract}

In the restoration of coal mine slopes, a high edaphic heterogeneity that determines important differences in the plant community is generated. A greater knowledge about the involved ecological processes is essential to guarantee the success of revegetation in degraded environments. This work evaluates the influence of the spatial variability of soil and topographic parameters on the distribution of vegetation on a hillside of a restored coal mine. The steep slope, located in Guardo (Palencia), was hydroseeded in October 2000. In the spring of 2011, six transects were established parallel to the topographic gradient, three in a grassland community and three in a scrubland community. Soils and vegetation were characterized along the topographic gradient. The vegetation is classified by families and by life cycle. A gradient in vegetation distribution was observed in response to altitude, increased water holding capacity and soil thickness, and a decrease in the easily oxidizable carbon / total carbon ratio. The pasture occupied the highest part of the slope and the scrub was in the lowest. The response of the vegetation along the altitudinal gradient was modeled. Woody and leguminous dominated the lower parts of the slope while the higher parts were occupied by grasses and annuals. The results suggest that the design of the hillsides for revegetation should consider the local topographic conditions.

Keywords: Topographic gradient, Restoration of degraded areas, Plant-soil relationship, Plant succession. 


\section{Introducción}

La extracción de carbón a cielo abierto en el norte de España ha modelado su paisaje desde hace más de dos siglos, y ha generado fuertes impactos ambientales en aproximadamente 5.000 hectáreas (López-Marcos et al., 2012). Por ello, la restauración de estas áreas es una necesidad urgente. Además, en España, la legislación obliga, desde 1982, a que todos los proyectos de explotación minera vayan acompañados de un plan de restauración del espacio natural afectado (R.D. 2994/1982), y establece las garantías necesarias para asegurar que dicho plan se cumpla. En los planes de restauración, para el remodelado del terreno y la revegetación deben tenerse en cuenta aspectos como el clima o las características edáficas del ambiente a restaurar para garantizar el éxito del procedimiento (R.D.975/2009). No obstante, en ocasiones las actuaciones propuestas no son suficientes para la correcta rehabilitación de los terrenos degradados, máxime en laderas, donde no se produce un desarrollo homogéneo de vegetación. A menudo este proceso de recuperación se ve dificultado por la total eliminación del suelo y la vegetación (Cooke and Johnson, 2002; Moreno-de las Heras et al., 2008).

La restauración ecológica, además de ser una técnica de recuperación de comunidades naturales, sirve como método de investigación básica en ecología. Se puede decir que la sucesión ecológica es el marco conceptual en el cual se basa la restauración ecológica (López-Marcos et al., 2017). El principal objetivo de la restauración ecológica es la creación de ecosistemas auto-suficientes a largo plazo, con vegetación semejante a la existente antes de la actividad extractiva (Grant, 2006), con buena cobertura vegetal (Dazy et al., 2008) y un suelo en pleno funcionamiento (Dölle and Schmidt, 2009; Moreno-de las Heras, 2009). Sin embargo, poco se sabe sobre cómo cambian los parámetros del nuevo suelo formado durante las diferentes fases de la revegetación de las minas de carbón (Alday et al., 2010). Numerosos estudios han puesto de manifiesto que las propiedades edáficas suelen presentar una distribución en forma de manchas (Ettema and Wardle, 2002) y, por lo tanto, la heterogeneidad espacial juega un papel importante en la estructura y la dinámica de los ecosistemas (Tilman, 1988), especialmente en ambientes mediterráneos, donde la distribución de la vegetación puede ser discontinua, causada por ejemplo por la presencia de patrones marcados en la distribución de algunas propiedades del suelo.

Por ello, el objetivo de este trabajo fue estudiar la influencia de la heterogeneidad edáfica y topográfica sobre la vegetación, en una ladera minera restaurada. Para ello, se evaluó la influencia del tipo de comunidad (pastizal y matorral) y su posición en la ladera (partes alta, media y baja) sobre la vegetación y las propiedades edáficas y su influencia en la dinámica sucesional.

\section{2. Material y métodos}

El área de estudio se sitúa cerca de la villa de Guardo, al noroeste de la provincia de Palencia, Castilla y León (Fig. 1). Se trata de una mina de carbón a cielo 
abierto, restaurada en octubre de 2000, dominada por pasto y matorral y ubicada en el monte Corcos (Guardo, lat 42 $47^{\prime} 18.4$ " N, long 450'59.2” W; 1110 m s.n.m.). Dentro de ella, se ha seleccionado una ladera de grandes dimensiones y fuerte pendiente $\left(12-35^{\circ}\right)$, orientada al sur, dominada por pastizal y matorral. El clima es Mediterráneo Sub-húmedo (Milder et al., 2013), con $973 \mathrm{~mm}$ de precipitación media anual, y una temperatura media anual de $9,2{ }^{\circ} \mathrm{C}$, para el período comprendido entre 1971 y 2007. Los suelos más extendidos entorno al área de estudio pertenecen al orden de los Inceptisoles con un régimen de humedad údico y un régimen de temperatura mésico (Soil-Survey-Staff, 2014).

La vegetación potencial se corresponde con la Serie Supra Carpetana occidental leonesa húmeda silicícola de Quercus pyrenaica (Holco mollis-Querceto pyrenaicae S.), concretamente en su faciación supramediterránea con Erica australis (RivasMartínez, 1987). Se trata de una vegetación de carácter húmedo-hiperhúmedo y se encuentra bien caracterizada por sus etapas sucesionales de piornales de Cytisus scoparius, Cytisus striatus y Genista florida subsp. polygaliphylla y, después de éstos, brezales de Erica australis con Genista tridentata (García y Jiménez, 2009).

Para alcanzar los objetivos planteados se muestrearon en dicha ladera, en primavera de 2011, seis transectos paralelos a la línea de pendiente, tres ubicados en el pastizal y tres en el matorral. En cada transecto se establecieron seis posiciones de muestreo consecutivas. Las 2 posiciones superiores de cada transecto se consideraron parte alta del pastizal o matorral respectivamente, las 2 siguientes, parte media y las 2 parcelas inferiores de cada transecto se consideraron parte baja (Fig. 1). En cada una de ellas se realizaron dos inventarios de vegetación, estimando la cobertura en porcentaje de la proyección vertical de cada una de las especies encontradas. La cobertura alcanzó en algunos casos valores superiores al 100\% debido a la superposición de la vegetación. El

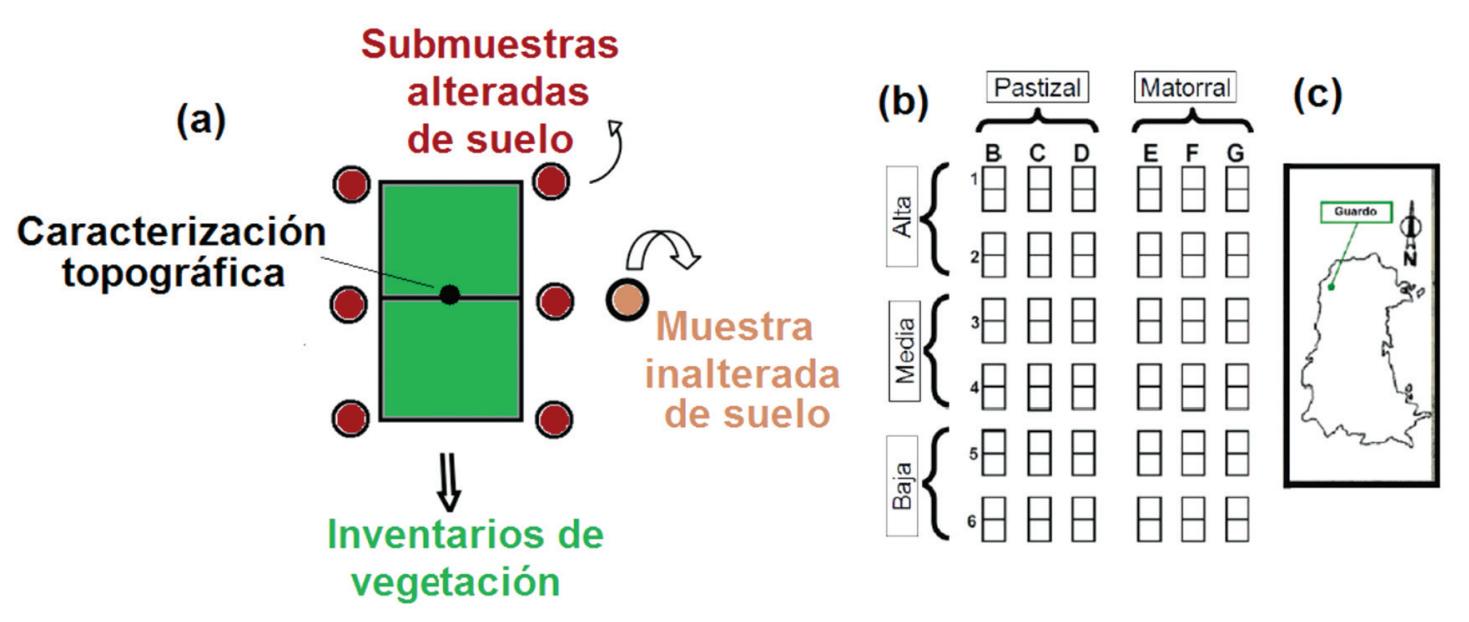

Figura 1. Diseño de muestreo. (a) Muestras tomadas en cada punto de muestreo: dos inventarios de vegetación, 6 submuestras alteraras de suelo, una muestra inalterada de suelo y un punto de caracterización topográfica; (b) Distribución de seis puntos de muestreo de vegetación, suelo y topografía a lo largo de cada uno de los seis transectos implementados para su estudio; y (c) Localización del área de estudio en la provincia de Palencia. 
tamaño de los inventarios varió debido a esta disparidad en la composición vegetal de los diferentes transectos: de $0,5 \times 0,5 \mathrm{~m}$ para pastizal y de $2 \times 2 \mathrm{~m}$ para matorral. Los tamaños estaban basados en estudios previos en la misma zona (Milder et al., 2013).

Además, en cada posición de cada transecto se realizó un muestreo edáfico (Fig. 1), tomando una muestra inalterada de suelo y una muestra de suelo alterado, compuesta de 6 submuestras tomadas en el entorno de cada par de inventarios de vegetación y homogeneizadas. Se analizaron diferentes parámetros edáficos tanto físicos como químicos: $\mathrm{pH}$, conductividad eléctrica, carbono fácilmente oxidable, fósforo asimilable, carbono y nitrógeno totales, ratio carbono fácilmente oxidable entre carbono total, $(\mathrm{CFO} / \mathrm{C})$, ratio carbono-nitrógeno $(\mathrm{C} / \mathrm{N}), \%$ de gruesos, textura, densidad real, densidad aparente, porosidad, capacidad de campo, coeficiente de marchitamiento, agua útil y capacidad de retención de agua (CRA). La información relativa a los métodos de análisis de los suelos puede consultarse en (López-Marcos, 2013).

La información de la cobertura de la vegetación se clasificó según su ciclo de vida: anuales, perennes o leñosas; o según la familia a la que pertenecían. Se identificaron tres familias dominantes: compuestas, gramíneas y leguminosas. El resto de familias se agruparon en otra categoría llamada "otras familias".

El tratamiento de los datos consistió en un análisis de redundancia (RDA) para ordenar los puntos de muestreo en función de las variables de vegetación (coberturas por ciclo de vida: anuales, perennes y leñosas, y cobertura por familias: compuestas, gramíneas, leguminosas y otras familias). Sobre el RDA se proyectó un análisis "envfit" para ajustar los vectores de las variables ambientales. El test de Monte Carlo (999 permutaciones; (Legendre and Legendre, 2003) se usó para evaluar la correlación máxima entre las variables ambientales en la ordenación RDA. Sólo se trazaron aquellas con una correlación significativa $(\mathrm{p}<0,05)$. Por último, se modelizó la respuesta de las variables de vegetación a la altitud mediante los modelos HOF. Los modelos HOF permiten describir la respuesta de la vegetación a gradientes ecológicos, en este caso gradientes de parámetros edáficos y topográficos (Lawesson and Oksanen, 2002). Se trata de un conjunto jerárquico de cinco modelos ordenados en sentido creciente de complejidad, desde el que describe la respuesta uniforme de las especies hasta el que describe una respuesta de campana de Gauss asimétrica (Huisman et al., 1993). El criterio de información de Akaike (AIC; Akaike, 1973) se utilizó para seleccionar el modelo de respuesta más adecuado. Todos estos análisis se realizaron en el entorno de R (versión 3.3.3; R-CoreTeam, 2015): paquetes "vegan" (Oksanen, 2016) y "eHOF” (Jansen and Oksanen, 2013), para los análisis RDA+envfit y modelos de HOF, respectivamente.

\section{Resultados}

El análisis RDA muestra una segregación de las parcelas en función de la vegetación. El eje 1 (RDA1; $\lambda_{1}=3,3$ ) explica el 46,7\% de la varianza, y junto con el eje 2 (RDA2; $\lambda_{2}=19,4$ ) explican el $66,1 \%$ (Fig. 2). 


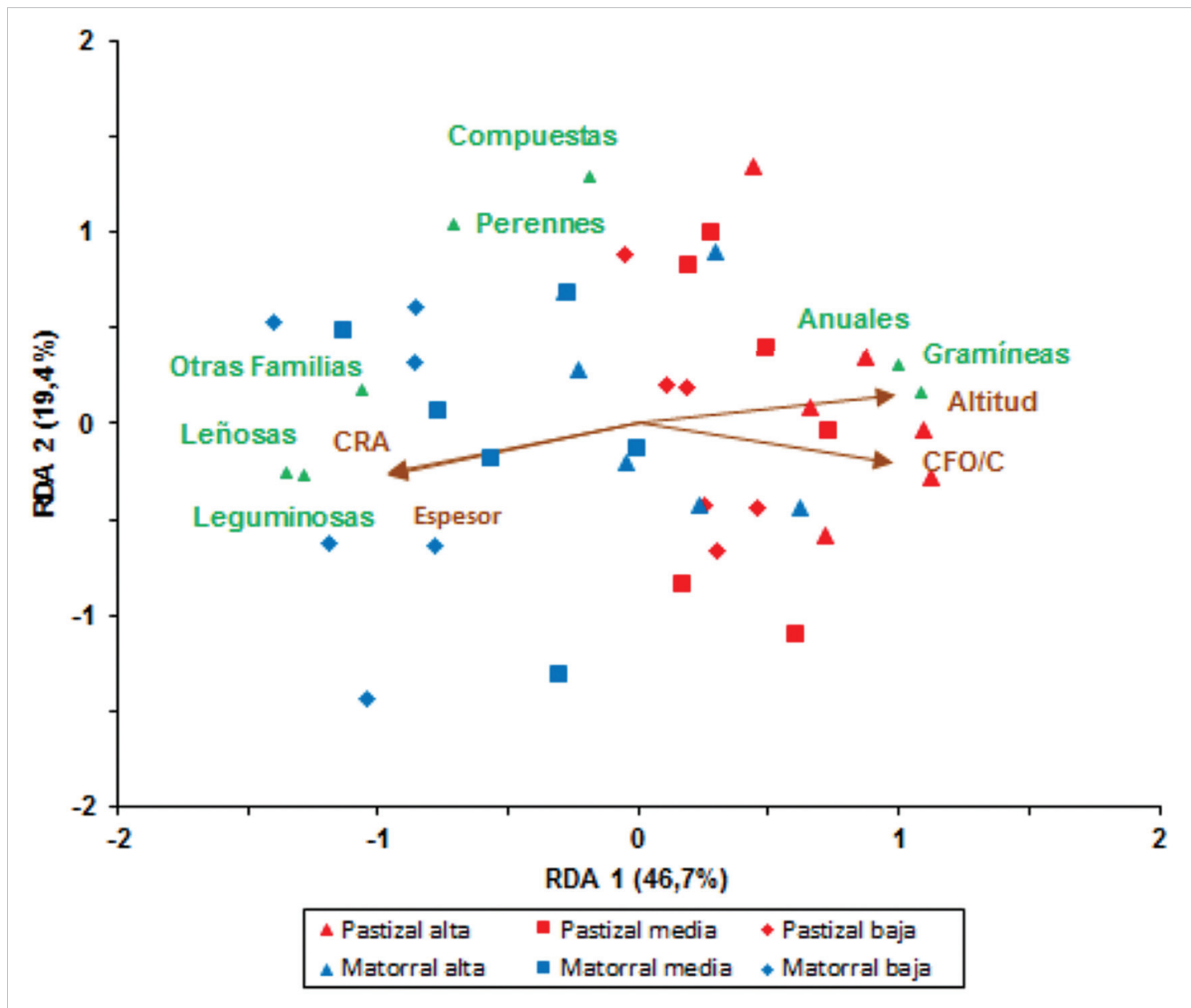

Figura 2. Triplot de los puntos de muestro (triángulos, cuadrados y rombos de azul y rojo), variables de vegetación (vectores verdes; por ciclo de vida: anuales, perennes y Leñosas; o por familias: compuestas, gramíneas, leguminosas y otras familias) y variables ambientales significativas del análisis "envfit" (vectores marrones: Altitud; CRA: capacidad de retención de agua; Espesor: espesor del suelo hasta la aparición del estéril; $\mathrm{CFO} / \mathrm{C}$ : ratio carbono fácilmente oxidable entre carbono total).

Se observa un gradiente de parcelas y las variables vegetación respecto al eje RDA1, situándose en el extremo derecho la mayoría de las parcelas que ocupan la parte alta del pastizal, donde predominan anuales y gramíneas. En la parte central del gradiente encontramos las parcelas que ocupan la posición baja del pastizal y alta del matorral, en las que destacan compuestas y perennes y finalmente, en el extremo izquierdo del eje RDA1 se sitúan las parcelas que ocupan la parte baja del matorral, que aparecen asociadas a las leguminosas y leñosas.

De todas las variables edáficas introducidas en el análisis "envfit" sólo fueron significativas: altitud $(r=0,89 ; \mathrm{p}<0,05), \mathrm{CRA}(\mathrm{r}=0,75 ; \mathrm{p}<0,05), \mathrm{CFO} / \mathrm{C}(\mathrm{r}=0,69 ; \mathrm{p}$ $<0,05)$ y espesor $(r=0,55 ; p<0,05)$. CRA y espesor del suelo aumentaron hacia la parte baja del matorral, mientras que altitud y $\mathrm{CFO} / \mathrm{C}$ aumentaron hacia la parte alta 
del pastizal (Fig. 2). Es decir, se describe un gradiente topográfico-sucesional asociado tanto a variables relacionadas con el agua en el suelo (CRA) como a variables relacionadas con la materia orgánica en el suelo $(\mathrm{CFO} / \mathrm{C})$. Por ello, se decidió modelizar la respuesta de la vegetación frente al gradiente topográfico-altitudinal.

La respuesta de cada variable de vegetación a lo largo del gradiente de altitud (Fig. 3) permite agrupar las variables en tres grupos: El primero incluye especies que muestran modelo I. Se trata de especies cuya cobertura permanece constante a lo largo de dicho gradiente (igualmente abundantes en las comunidades de pastizal y matorral) y que no aparecen representadas en la figura 3; es el caso de perennes y compuestas. El segundo grupo incluye variables que muestran modelo II con tendencia creciente a medida que aumenta la altitud hacia el extremo izquierdo, es decir son más abundantes en las partes altas del pastizal, como es el caso de anuales y gramíneas. El tercer y último grupo incluye las variables que muestran modelo II con tendencia creciente a medida de disminuye la altitud, es decir, más abundantes en la comunidad de matorral, como es el caso de leñosas y leguminosas.

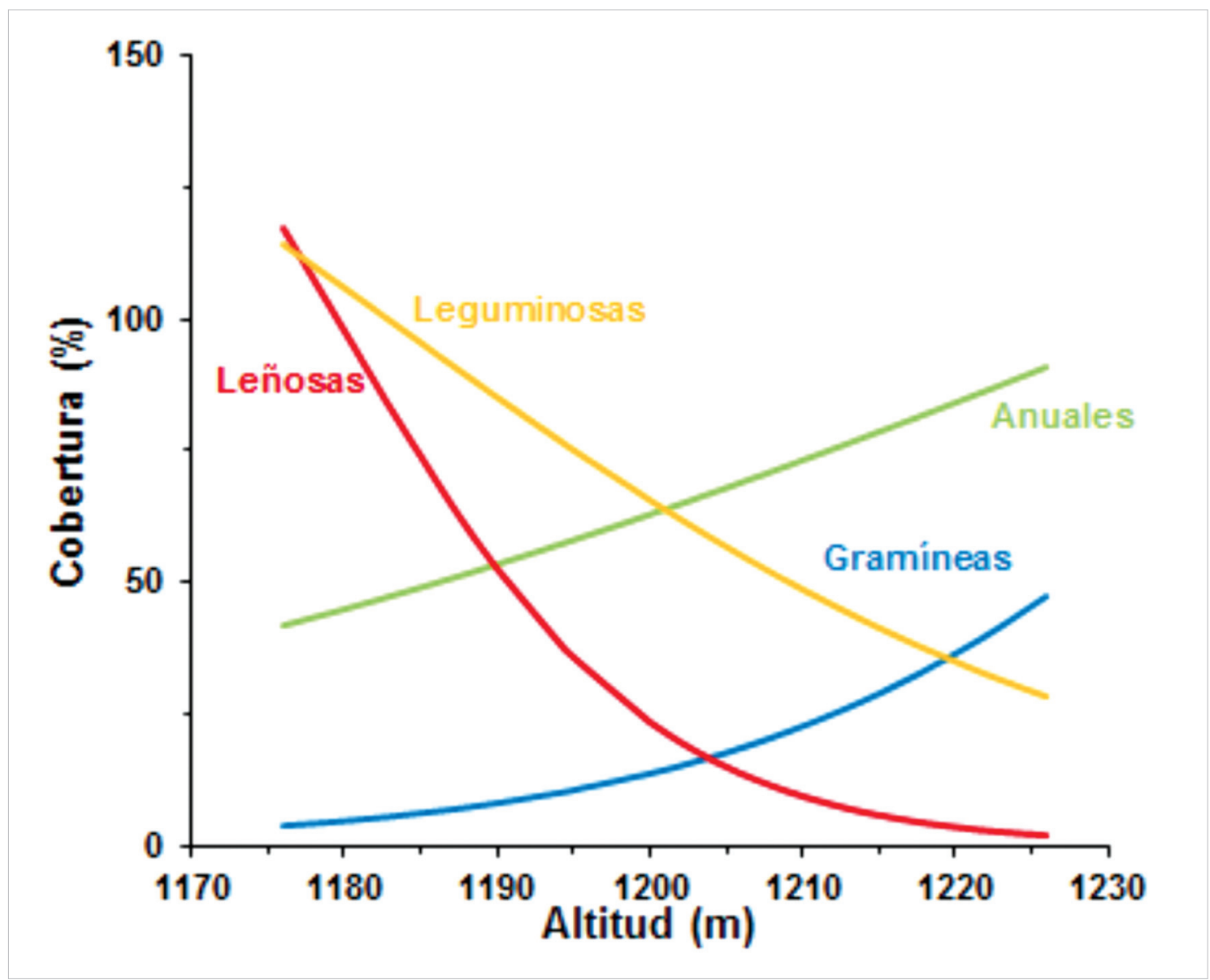

Figura 3. Modelos de HOF de las variables de vegetación a lo lardo del gradiente topográfico-altitudinal identificado. La cobertura alcanzó en algunos casos valores superiores al $100 \%$ debido a la superposición de la vegetación. 


\section{Discusión}

Los resultados ponen de manifiesto la existencia de un gradiente edáfico-topográfico, asociado al eje 1 del RDA, en la distribución de las comunidades vegetales a lo largo de la ladera restaurada; desde comunidades pascícolas dominadas por plantas anuales y gramíneas, hasta las comunidades de piornal más maduras dominadas por plantas leñosas y leguminosas. Estas diferencias específicas a nivel local pueden deberse a diferencias en la disponibilidad de propágulos (Bakker and Berendse, 1999; Pywell et al., 2002), al tipo de restauración (White and Jentsch, 2004) o a la estocasticidad ambiental (Bradshaw, 1983). En nuestro caso, se ha constatado que la CRA es la variable edáfica más influyente en la distribución de las especies en la ladera restaurada, revelándose como una de las variables más relacionadas con la dinámica de la vegetación hacia etapas más maduras. La CRA presenta los valores más altos en la parte baja de la ladera, donde la cobertura de leguminosas leñosas es más alta; y los valores más bajos en la parte alta del pastizal, donde se asienta un pastizal dominado por gramíneas anuales (López-Marcos et al., 2019). Este gradiente sucesional también ha sido descrito por Kardol et al. (2005) y (Moreno-de las Heras et al., 2008). Uno de los factores limitantes para el crecimiento vegetal es la baja capacidad para almacenar agua del suelo (Barnhisel et al., 1986). Por ello el incremento de la CRA hacia las partes bajas de la ladera parece favorecer el incremento de la cobertura vegetal leñosa, ya que en la parte baja es donde dominan las leguminosas arbustivas con coberturas de casi el 100\%. Este aspecto es muy relevante en el ámbito de la restauración puesto que la cubierta vegetal juega un papel importante en la estabilización de los sistemas alterados (Moreno-de las Heras, 2009).

Condron and Newman (1998) indicaron que la biomasa vegetal en algunas etapas de sustitución del bosque contiene gran cantidad de lignina que permanece en la hojarasca que llega al suelo. La mayor presencia de lignina reduce la tasa de descomposición de la materia orgánica del suelo por los microorganismos (Clark and Paul, 1970), dando lugar a una materia orgánica edáfica menos lábil (Chabrerie et al., 2003). Este aspecto se corrobora con nuestros resultados, ya que el porcentaje de carbono fácilmente oxidable frente al carbono total es significativamente mayor en el pastizal que en el matorral, indicando una materia orgánica más recalcitrante en el caso del suelo bajo matorral que bajo pastizal. Monterroso et al. (2004), en su estudio sobre fijación del C en el suelo a lo largo de 10 años, encuentran que la incorporación de carbono al suelo es rápida en el período inicial ( $0-4$ años) pero lenta después (4-10 años). Esto puede estar relacionado con la labilidad de la materia orgánica que se incorpora. En las etapas iniciales la incorporación de materia orgánica es debida principalmente a hojas y tallos verdes de la vegetación pascícola y más tarde la incorporación de materia orgánica es debida principalmente a hojas y tallos leñosos de las plantas propias de etapas más maduras y de degradación más lenta.

Finalmente, se observó una segregación en la abundancia de la vegetación en función de su ciclo de vida o de la familia a que pertenecen a lo largo de la ladera restaurada, que responde principalmente al gradiente topográfico (altitud) y de dis- 
ponibilidad hídrica (CRA) que, a su vez, determinan un gradiente en la cantidad de materia orgánica de los suelos $(\mathrm{CFO} / \mathrm{C})$. La vegetación cuya cobertura permanece constante a lo largo del gradiente son, en su mayoría herbáceas propias de la flora arvense y ruderal (Pujadas Salvá, 1986), que aparecen en muchos tipos de ambientes sin ser muy exigentes con sus requerimientos, como es el caso de perenes y compuestas que mostraron una respuesta monótona al gradiente estudiado.

La vegetación cuya cobertura disminuye desde las partes altas (pastizal) a las bajas (matorral) de la ladera, es decir, desde valores altos de la ratio $\mathrm{CFO} / \mathrm{C}$ y bajos de la CRA, son herbáceas anuales y gramíneas propias de pastos xerofíticos, sobre suelos con poca humedad edáfica (García et al., 1991). Finalmente, la vegetación cuya cobertura aumenta hacia las partes bajas de la ladera, dominadas por matorral, donde se registran bajos valores de la ratio $\mathrm{CFO} / \mathrm{C}$ y altos de la CRA son, en su mayoría, leguminosas leñosas características de la comunidad de matorrales nanofanerofíticos dominados por leguminosas arbustivas pertenecientes a la asociación Cytiso scoparii-Genistetum polygaliphyllae, que es una de las primeras etapas de sustitución de la etapa clímax de la zona de estudio (Holco mollis-Quercetum pyrenacicae S.) (Navarro-Andrés y Valle-Gutiérrez, 1987).

Estos resultados deben hacernos reflexionar acerca de las actuales técnicas de restauración de espacios degradados. Pequeñas diferencias en la topografía o en el sustrato de partida se revelan como limitantes de importantes procesos ecológicos, que tras varios años determinan diferentes trayectorias en la dinámica sucesional y en los patrones de respuesta individual de las especies.

\section{Conclusiones}

Nuestros resultados indican que la capacidad de retención de agua de los suelos (CRA) es una de las variables más relacionadas con la dinámica de la vegetación hacia etapas más maduras. Las especies propias de las etapas más avanzadas de la sucesión, representadas en este estudio por leguminosas leñosas, ocupan antes la parte baja de la ladera restaurada, que se corresponden con las comunidades de matorral donde la CRA es mayor. Valores más elevados de la fracción más lábil del carbono (CFO/C) están asociados a etapas sucesionales tempranas como pastizales. La sucesión vegetal avanza a diferente velocidad en las diferentes posiciones de la ladera. Comunidades maduras ocupan las posiciones bajas, mientras que las posiciones altas están dominadas por comunidades pioneras. Las especies pioneras son sustituidas por especies propias de etapas más maduras a lo largo del gradiente topográfico-sucesional descrito en la ladera restaurada.

\section{Agradecimientos}

Esta investigación ha sido parcialmente financiada por los proyectos VA042A10-2 y VA035G18 de la Junta de Castilla y León concedidos a C. Martínez-Ruiz. 


\section{Bibliografía}

Akaike, H.; 1973. Information theory as an extension of the maximum likelihood principle, In: Brillinger, D.; Gani, J.; Hartigan, J.; (eds.), Second International Symposium on Information Theory. Akademiai Kiado, Budapest (Hungary), pp. 267-281. https://doi. org/10.1007/978-0-387-98135-2

Alday, J.G.; Marrs, R.H.; Martínez-Ruiz, C.; 2010. The importance of topography and climate on short-term revegetation of coal wastes in Spain. Ecol. Eng. 36, 579-585. https://doi.org/10.1016/j.ecoleng.2009.12.005

Bakker, J.P.; Berendse, F.; 1999. Constraints in the restoration of ecological diversity in grassland and heathland communities. Trends Ecol. Evol. 14, 63-68. https://doi.org/ 10.1016/S0169-5347(98)01544-4

Barnhisel, R.I.; Powell, J.L.; Hines, D.H.; 1986. Changes in Chemical and Physical Properties of Two Soils in the Process of Surface Mining. Proc. Am. Soc. Surf. Min. Reclam. 17-19, 313-322. https://doi.org/10.21000/JASMR87010313

Bradshaw, A.D.; 1983. The Reconstruction of Ecosystems. J. Appl. Ecol. 20, 1-17. https:// doi.org/0021-8901/0400-0001

Chabrerie, O.; Laval, K.; Puget, P.; Desaire, S.; Alard, D.; 2003. Relationship between plant and soil microbial communities along a successional gradient in a chalk grassland in north-western France. Appl. Soil Ecol. 24, 43-56. https://doi.org/10.1016/S0929-1393 (03)00062-3

Clark, F.E.; Paul, E.A.; 1970. The microflora of grassland. Adv. Agron. 22, 375-435. https: //doi.org/10.1016/S0065-2113(08)60273-4

Condron, L.M.; Newman, R.H.; 1998. Chemical nature of soil organic matter under grassland and recently established forest. Eur. J. Soil Sci. https://doi.org/10.1046/j.13652389.1998.4940597.x

Cooke, J.A.; Johnson, M.S.; 2002. Ecological restoration of land with particular reference to the mining of metals and industrial minerals: A review of theory and practice. Environ. Rev. 10, 41-71. https://doi.org/10.1139/a01-014

Dazy, M.; Jung, V.; Férard, J.F.; Masfaraud, J.F.; 2008. Ecological recovery of vegetation on coke-factory soil: role of plant antioxidant enzymes and possible implications in site restoration. Chemosphere 74, 57-63. https://doi.org/10.1016/j.chemosphere.2008.09. 014

Dölle, M.; Schmidt, W.; 2009. Impact of tree species on nutrient and light availability: Evidence from a permanent plot study of old-field succession. Plant Ecol. 203, 273 -287. https://doi.org/10.1007/s11258-008-9547-2

Ettema, C.H.; Wardle, D.A.; 2002. Spatial soil ecology. Trends Ecol. Evol. 17, 177-183. https://doi.org/10.1016/S0169-5347(02)02496-5

García, I.; Jiménez, P.; 2009. 9230 Robledales de Quercus pyrenaica y robledales de Quercus robur y Quercus pyrenaica del Noroeste ibérico. In: Bases ecológicas preliminares para la conservación de los tipos de hábitat de interés comunitario en España. Ministerio de Medio Ambiente, y Medio Rural y Marino., Madrid (España).

García, R.; Moro, A.; Pérez-Pinto, J.E.; Pérez-Pinto, T.; Calleja, A.; 1991. Composición botánica y producción de prados permanentes de montaña. Pastos 20-21, 19-49.

Grant, C.D.; 2006. State-and-transition successional model for bauxite mining rehabilitation 
in the Jarrah forest of Western Australia. Restor. Ecol. 14, 28-37. https://doi.org/10.11 11/j.1526-100X.2006.00102.x

Huisman, J.; Olff, H.; Fresco, L.F.M.; 1993. A hierarchical set of models for species response analysis. J. Veg. Sci. CN - 3619 4, 37-46. https://doi.org/10.2307/3235732

Jansen, F.; Oksanen, J.; 2013. How to model species responses along ecological gradients Huisman-Olff-Fresco models revisited. J. Veg. Sci. 24, 1108-1117. https://doi.org/10.1 111/jvs. 12050

Kardol, P.; Bezemer, T.M.; Van Der Wal, A.; Van Der Putten, W.H.; 2005. Successional trajectories of soil nematode and plant communities in a chronosequence of ex-arable lands. Biol. Conserv. 126, 317-327. https://doi.org/10.1016/j.biocon.2005.06.005

Lawesson, J.E.; Oksanen, J.; 2002. Niche characteristics of Danish woody species as derived from coenoclines. J. Veg. Sci. 13, 279-290. https://doi.org/10.1111/j.1654-1103.2002. tb02048.x

Legendre, P.; Legendre, L.; 2003. Numerical Ecology, Developments in Environmental Modelling. Elservier, Amsterdam (Nederland). https://doi.org/10.1017/CBO97811 07415324.004

López-Marcos, D.; 2013. Variabilidad edafo-topográfica y de estructura de la vegetación de comunidades vegetales asentadas sobre estériles de carbón. Tesina de Licenciatura. University of León.

López-Marcos, D.; Turrión, M.B.; Martínez-Ruiz, C.; 2019. Linking soil variability with plant community composition along a mine-slope topographic gradient: Implications for restoration. Ambio. https://doi.org/10.1007/s13280-019-01193-y

López-Marcos, D.; Turrión, M.B.; Martínez-Ruiz, C.; 2017. Influencia de la variabilidad edáfica en la comunidad vegetal asentada en una ladera minera: Implicaciones para la restauración. In: SECF (ed.), 7th Spanish Forestry Congress. SECF, Plasencia, Cáceres (Spain).

López-Marcos, D.; Turrión, M.B.; Martínez-Ruiz, C.; 2012. Influencia de la heterogeneidad edáfica y de la topografía en la composición de especies vegetales sobre escombreras mineras de carbón del norte de España. In: Martínez-Ruiz, C.; Lario-Leza, F.; Fernandez-Santos, B.; (eds.), Avances en la restauración de sistemas forestales. Técnicas de implantación. AEET - SECF, Palencia (Spain), pp. 215-223.

Milder, A.I.; Fernandez-Santos, B.; Martínez-Ruiz, C.; 2013. Colonization Patterns of Woody Species on Lands Mined for Coal in Spain: Preliminary Insights for Forest Expansion. L. Degrad. Dev. 24, 39-46. https://doi.org/10.1002/ldr.1101

Monterroso, C.; Gil Bueno, A.; Pérez Varela, S.; Macías, F.; 2004. Restauración de suelos de mina: contribución a la fijación de carbono en el ecosistema terrestre. Edafología 11, $135-148$.

Moreno-de las Heras, M.; 2009. Development of soil physical structure and biological functionality in mining spoils affected by soil erosion in a Mediterranean-Continental environment. Geoderma 149, 249-256. https://doi.org/10.1016/j.geoderma.2008.12.003

Moreno-de las Heras, M.; Nicolau, J.M.; Espigares, T.; 2008. Vegetation succession in reclaimed coal-mining slopes in a Mediterranean-dry environment. Ecol. Eng. 34, 168178. https://doi.org/10.1016/j.ecoleng.2008.07.017

Navarro-Andrés, F.; Valle-Gutiérrez, C.J.; 1987. Castilla y León. In: Peinado Lorca, M.Y., Rivas Martínez, S. (eds.), La Vegetación de España. Aula abierta, Madrid (Spain), pp. $117-161$. 
Oksanen, J.; 2016. Vegan: an introduction to ordination 1-12.

Pujadas Salvá, A.; 1986. Flora arvense y ruderal de la provincia de Córdoba. Universidad de Córdova.

Pywell, R.F.; Bullock, J.M.; Hopkins, A.; Walker, K.J.; Sparks, T.H.; Burke, M.J.W.; Peel, S.; 2002. Restoration of species rich grassland on arable land: assessing the limiting processes using a multi site experiment. J. Appl. Ecol. 39, 294-309. https://doi.org/ 10.1046/j.1365-2664.2002.00718.x

R-Core-Team, 2015. R Studio: Integrated development environment for R.

R.D.2994/1982, 1982. Real Decreto 2994/1982 de 15 de octubre, sobre restauración de espacio natural afectado por actividades mineras. BOE $\mathrm{n}^{\circ} 274$ de 15 de noviembre de 1982, páginas 31246 a 31247.

R.D.975/2009, 2009. Real Decreto 975/2009, de 12 de junio, sobre gestión de los residuos de las industrias extractivas y de protección y rehabilitación del espacio afectado por actividades mineras. BOE n ${ }^{\circ} 143$, de 13 de junio de 2009, páginas 49948 a 49993.

Rivas-Martínez, S.; 1987. Memoria del mapa de series de vegetación de España. Serie Técnica, Madrid (Spain).

Soil-Survey-Staff, 2014. Keys to Soil Taxonomy, 12 th. ed. USDA-Natural Resources Conservation Service, Washington, DC (USA). https://doi.org/10.1109/TIP.2005.854494

Tilman, D.; 1988. Plant Strategies and the Dynamics and Structure of Plant Communities. Princeton University Press, Princeton (USA).

White, P.S.; Jentsch, A.; 2004. Disturbance, succession, and community assembly in terrestrial plant communities. In: Temperton, V.M., Hobbs, R.J., Nuttle, T., Halle, S. (Eds.), Assembly Rules and Restoration Ecology. Island Press, Washington, DC (USA), pp. 342-366. 